Collection SFN 12 (2011) 105-121

(C) Owned by the authors, published by EDP Sciences, 2011

DOI: $10.1051 / \mathrm{sfn} / 201112006$

\title{
Numerical simulations and magnetism
}

S. Petit

Institut Rayonnement Matière de Saclay, Laboratoire Léon Brillouin, CEA-CNRS UMR 12, 91190 Gif-sur-Yvette, France

\section{INTRODUCTION}

Owing to recent developments in numerical and computer science, numerical simulations have become an important trend in physics and a useful tool to design and explain experiments. In the context of this school, organized by the French neutron scattering society, SFN, this lecture presents two examples of numerical simulations aiming at computing the spin-spin correlation function measured by neutron scattering. The first is an introduction to the linear spin wave theory. The second offers some aspects of Monte Carlo simulations of the classical spin dynamics. To serve as a prelude of these two examples, we start with a brief introduction to neutron techniques and to their use in measuring the spin dynamics in magnets.

\section{NEUTRON AND SPIN DYNAMICS}

In a scattering process, the incident neutrons, with a wavevector $\vec{k}_{i}$, are scattered under a solid angle $\Omega$ with a final wavevector $\vec{k}_{f}$. As the neutron's wavefunction is a plane wave, its energy $E$ directly depends on its wavenumber $k$ :

$$
E=\frac{\hbar^{2} k^{2}}{2 M_{n}}
$$

where $M_{n}$ is the neutron's mass. Simultaneously, the target is excited or decays from an initial state labelled $|\lambda\rangle$ with energy $E_{\lambda}$ to the final state $\left|\lambda^{\prime}\right\rangle$ with energy $E_{\lambda^{\prime}}$. The conservation laws impose (we forget about the factor $\frac{\hbar^{2}}{2 M_{n}}$ for convenience):

$$
\left\{\begin{array}{l}
\vec{Q}=\vec{k}_{i}-\vec{k}_{f} \\
E_{\lambda}+k_{i}^{2}=E_{\lambda^{\prime}}+k_{f}^{2}
\end{array}\right.
$$

and the energy balance is defined as:

$$
\omega=k_{i}^{2}-k_{f}^{2}=E_{\lambda^{\prime}}-E_{\lambda}
$$

It is worth noting that neutrons with different final energies can be scattered in the same scattering angle (see figure 1). In other words, the scattered beam contains neutrons of different energies, spreading from $E_{f}=0$ (the incident neutron gives its whole energy) to infinity (in that case, the sample gives some energy to the neutron). Meanwhile, each individual process is described by a cross section that depends on the final energy and on the solid angle $\Omega$. The calculation of the magnetic cross section is beyond the scope of this article; however, it can be shown that it is proportional to a spin-spin correlation

This is an Open Access article distributed under the terms of the Creative Commons Attribution-Noncommercial License 3.0, which permits unrestricted use, distribution, and reproduction in any noncommercial medium, provided the original work is properly cited. 


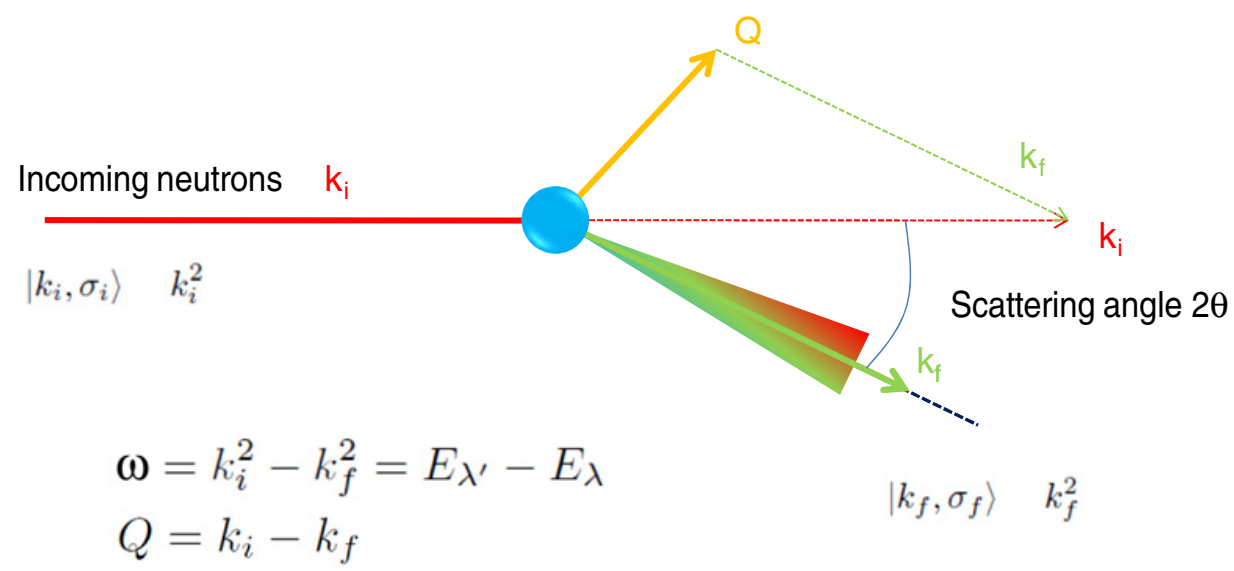

Figure 1. Schematic diagram of a scattering process.

function:

$$
\begin{aligned}
\frac{\partial^{2} \sigma}{\partial \Omega \partial E_{f}}(Q, \omega)= & \frac{k_{f}}{k_{i}}\left(\gamma r_{o}\right)^{2} \sum_{m, n, i, j} e^{i Q\left(R_{m}+u_{i}-R_{n}-u_{j}\right)} f_{i}(Q) f_{j}(Q)^{*} e^{-\left(W_{i}(Q)+W_{j}(Q)\right)} \\
& \times \int d t e^{-i \omega t} \sum_{a, b=x, y, z}\left(\delta_{a, b}-\frac{Q^{a} Q^{b}}{Q^{2}}\right)\left\langle S_{m, i}^{\alpha} S_{n, j}^{\beta}(t)\right\rangle
\end{aligned}
$$

In this expression, the position of the $i$ th ion in the unit cell $m$ is defined by $R_{m}+u_{i}, \gamma r_{o}$ is the classical radius of an electron, $W_{i}$ the Debye-Waller factor and $f_{i}$ is the magnetic form factor of the $i$ th ion. The former is due to the fact that the electrons are bound to ions that keep moving because of thermal fluctuations, and the latter to the fact that the electrons occupy orbitals with a finite spatial extension. The factor $\left(\delta_{a, b}-\frac{Q^{a} Q^{b}}{Q^{2}}\right)$ is due to the dipolar nature of the magnetic interaction between neutron and electrons. The physics is included in the spin-spin correlation functions:

$$
\left\langle S_{m, i}^{\alpha} S_{n, j}^{\beta}(t)\right\rangle
$$

which contain valuable information on the arrangement of the spins and of their dynamics.

\subsection{Diffraction}

Let us now examine in some details the scattering process. First, we shall consider a diffraction experiment. In that case, the detector is positioned in the scattered beam, selecting a scattering angle denoted by $2 \theta$. Together with the monochromator which selects the incident neutron energies, we have the two fundamental elements of a neutron diffractometer. It turns out that the elastic processes, in which the neutrons keep their energy, do have the largest cross section. As in that case $k_{i}=k_{f}$, we have:

$$
\begin{aligned}
Q^{2} & =k_{i}^{2}+k_{f}^{2}-2 k_{i} k_{f} \cos 2 \theta \\
& =2 k_{i}^{2}(1-\cos 2 \theta) \\
& =4 k_{i}^{2} \sin ^{2} \theta \\
Q & =2 k_{i} \sin \theta
\end{aligned}
$$


(a)

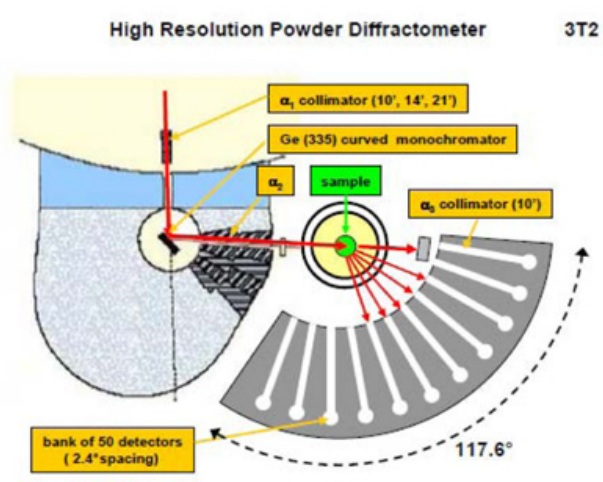

(b)

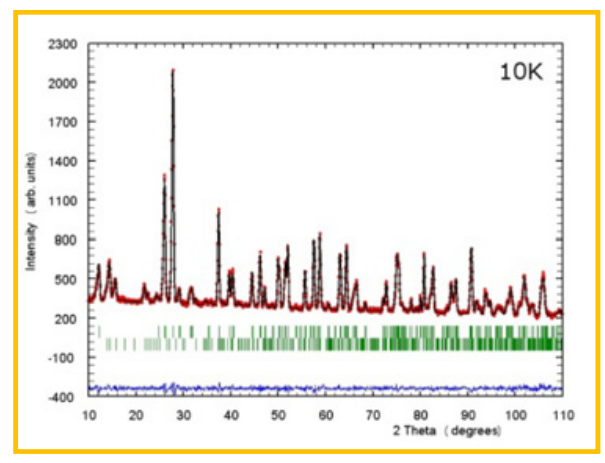

Figure 2. (a) Schematic diagram of a powder diffractometer. (b) Example of a diffraction profile along with the refinement (in red).

Substituting the lattice spacing $d=2 \pi / Q$ and incident wavelength of the neutron $\lambda=\frac{2 \pi}{k_{i}}$ in the previous equations, we finally obtain the Bragg law:

$$
\lambda=2 d \sin \theta
$$

This formula tells us that if we record the number of neutrons scattered in the angle $2 \theta$, simply by moving the detector step by step, we expect non zero intensity each time the Bragg law is satisfied. We thus collect the different $d$ spacings of the sample. In real life, of course, we do not move the detector step by step because it would be time consuming. Instead, we use a bank of detector that cover a wide range of angles. As a result, we have in one shot a scattering profile, as shown in figure 2, which remains to be fitted to determine the magnetic or crystalline structure. It can be shown that the elastic cross section is proportional to the structure factor:

$$
\sum_{m, n, i, j} e^{i Q\left(R_{m}+u_{i}-R_{n}-u_{j}\right)} f_{i}(Q) f_{j}(Q)^{*} e^{-\left(W_{i}(Q)+W_{j}(Q)\right)} \sum_{a, b}\left(\delta_{a, b}-\frac{Q^{a} Q^{b}}{Q^{2}}\right)\left\langle S_{m, i}^{\alpha}\right\rangle\left\langle S_{n, j}^{\beta}\right\rangle
$$

The Rietweld refinement of the peak positions and of their intensity (with for example the Fullprof-suite) allows generally to determine the atomic positions and directions of the magnetic moments within the unit cell.

As explained, elastic processes have the largest cross section, but we should not forget about the neutrons that have undergone an inelastic scattering. These processes contribute to the intensity and may have a significant contribution. In most cases, it looks like a diffuse signal, a bump roughly centred around the scattering angle $2 \theta$ where the magnetic Bragg peak rises in the ordered state.

\subsection{Inelastic scattering}

Let us now move to inelastic scattering. For a given $Q$ and $\omega$, it is in principle possible to obtain a measure of the spin-spin correlation function:

$$
\int d t e^{-i \omega t}\left\langle S_{m, i}^{\alpha} S_{n, j}^{\beta}(t)\right\rangle
$$

Actually, measuring the corresponding cross section requires to select a specific scattering process with a given momentum transfer $\vec{Q}=\vec{k}_{i}-\vec{k}_{f}$ and with incident and final energies so that $\omega=E_{i}-E_{f}$. 
(a) Triple axis
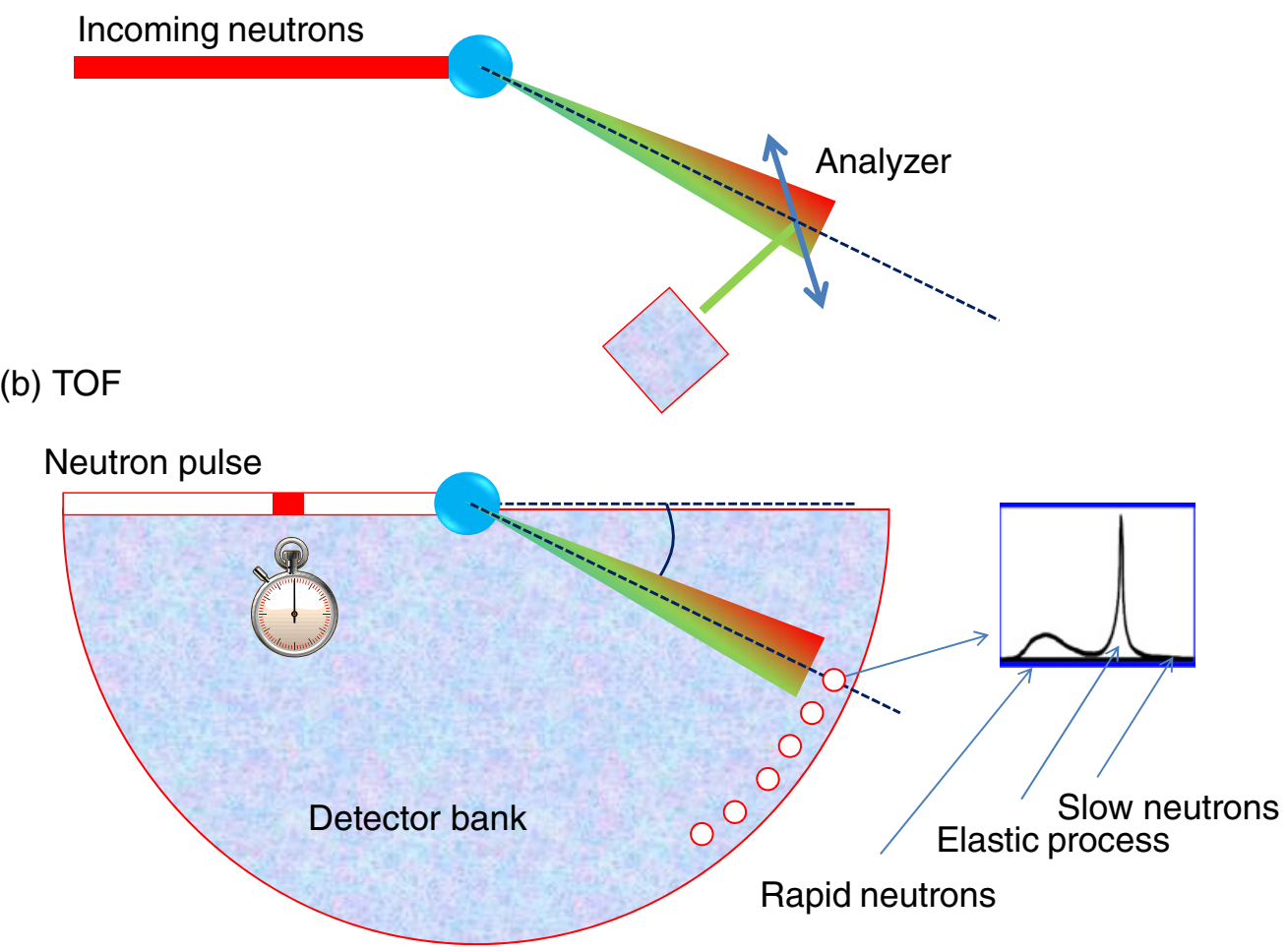

Figure 3. (a) Schematic diagram of a triple axis spectrometer and (b) of a time of flight spectrometer.

Using the conservation laws, we can show that the modulus of $\vec{Q}$ imposes the scattering angle:

$$
Q^{2}=2 k_{i}^{2}-\omega-2 k_{i} \sqrt{k_{i}^{2}-\omega} \cos 2 \theta
$$

Note that there are some cases where both the energy and momentum conservation cannot be fulfilled simultaneously: the $\left(\vec{Q}, \vec{k}_{i}, \vec{k}_{f}\right)$ scattering triangle cannot close (see figure 1). Accordingly, the cross section for the corresponding $(\vec{Q}, \omega)$ cannot be measured. It should be also noted that if the sample is a single crystal, we need to rotate it around the vertical axis, so that the desired $\vec{Q}$ vector of the reciprocal lattice coincides with the $\vec{Q}$ vector imposed by the scattering geometry (see figure 4). Of course, this is unnecessary if the sample is a powder, as in that case the powder average eliminates any angular $\vec{Q}$ dependence.

On a triple axis spectrometer, the choice of $E_{f}$ is easily done, taking advantage of the Bragg law, with an analyzer, which is nothing but a monochromator installed in the scattered beam.

TOF spectrometers follow a different strategy, analyzing the final energy of the neutron owing to their time of flight $t_{\mathrm{TOF}}$. The device that analyzes the final energy is simply a chronometer. As a function of time, a bank of detectors records for a set of scattering angles simultaneously, first neutrons that have been accelerated by the sample, then the elastic processes, and finally the neutrons that gave part of their energy to the sample. To be efficient, it is however necessary to pulse the incoming neutron beam. The time between two pulses must be chosen carefully to avoid overlapping of the data. It is also necessary to reduce the data to convert the intensities recorded as a function of $\left(t_{\mathrm{TOF}}, 2 \theta\right)$ to the $(Q, \omega)$ variables.

This was only a brief introduction, aiming at a quick description of the basis of magnetic neutron scattering. We refer the reader to other lectures for further details, and especially to obtain more specific 
(a)

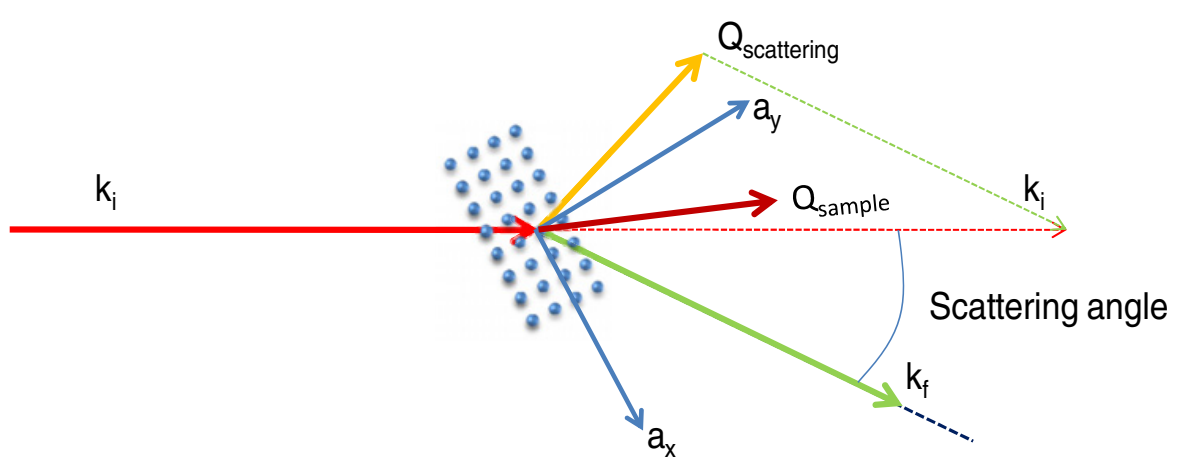

(b)

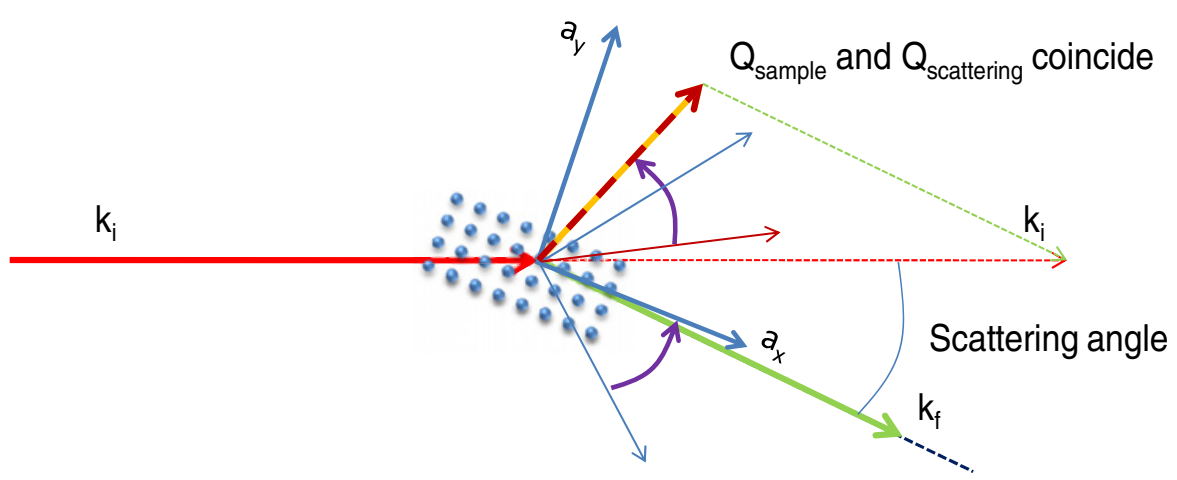

Figure 4. (a) In an inelastic scattering experiment, the incident and final energies of the neutron are chosen so that $\omega=E_{i}-E_{f}$. The scattering angle imposes the modulus of the wavevector $\vec{Q}$ owing to the formula $Q^{2}=2 k_{i}^{2}-\omega-2 k_{i} \sqrt{k_{i}^{2}-\omega} \cos 2 \theta$. This sets the components of the wavevector $\vec{Q}=\vec{k}_{i}-\vec{k}_{f}$ in the lab geometry. (b) Meanwhile, the reciprocal lattice of a single crystal sample defines its own frame. To let coincide $\vec{Q}$, defined by $\vec{k}_{i}$ and $\vec{k}_{f}$ and denoted by $\vec{Q}_{\text {scattering }}$, and the desired reciprocal lattice $\vec{Q}$ vector, denoted by $\vec{Q}_{\text {sample }}$, it is necessary to rotate the sample.

information about the nuclear and magnetic cross section as well as on the design of the spectrometers and calculation of the resolution function.

\section{SPIN WAVE THEORY}

We now turn to the simulation of the spin dynamics, and first examine the spin wave theory, which is one of the milestones in magnetism [1-8]. While developed in the 1950's, it continues to be of fundamental importance. It is based on the Heisenberg Hamiltonian:

$$
H=\sum_{m, n, i, j} \vec{S}_{m, i} J_{m, i, n, j} \vec{S}_{n, j}+\sum_{m, i} D_{i}\left(\vec{S}_{m, i} \cdot \vec{n}_{i}\right)^{2}
$$

which takes into account exchange couplings $J_{m, i, n, j}$ between spins located at sites $(m, i)$ and $(n, j)$. Here, $i$ is an index over the spin position within the unit cell (denoted by the index $m$ ). The second term is a single ion anisotropy term. As we want to minimize the energy, it is clear that if $D$ is negative, $\vec{n}_{i}$ is a local easy-axis direction (at site $i$ ): the spins prefer to align along this direction. In contrast, if $D$ is positive, $\vec{n}_{i}$ is perpendicular to an easy-plane, and the system gains energy if the spins lie down into that plane. 

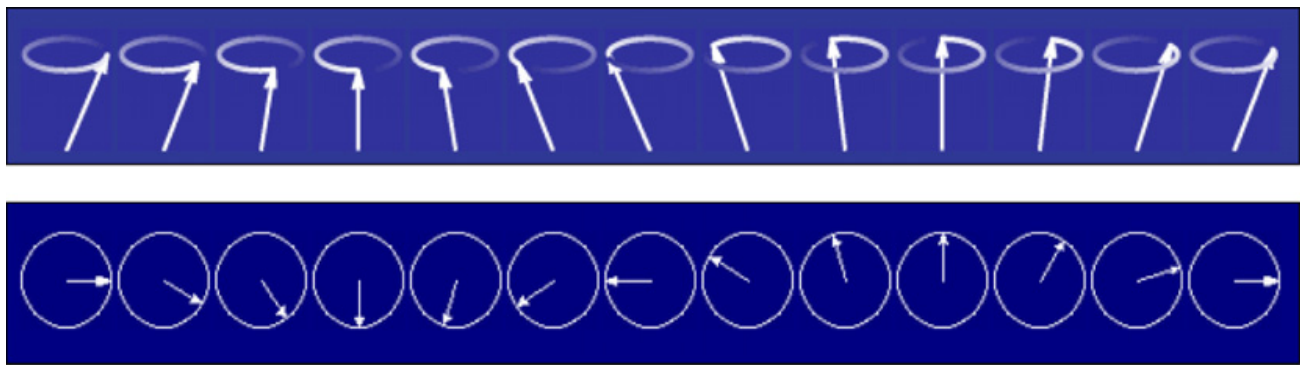

Figure 5. Schematic diagram of a spin wave in a ferromagnet.

\subsection{The hypotheses}

The two hypotheses of the spin wave theory are the following:

- First, the molecular field induces a phase transition towards a magnetically ordered state. Accordingly, we shall define $N$ local frames, attached to the different ions of the magnetic unit cell, denoted $\left(e_{i}^{1}, e_{i}^{2}, e_{i}^{3}\right)$, where $e_{i}^{3}$ corresponds to the direction of the $i$ th ordered moment. Because of translation symmetry, it is clear that these bases do not depend on the $m$ indices. We shall also define a set of matrices, connecting the real Cartesian space to the local frames:

$$
S_{i}^{\alpha=x, y, z}=\sum_{\mu=1,2,3} R_{i}^{\alpha, \mu} S_{i}^{\mu}
$$

as well as the useful vectors:

$$
\begin{aligned}
z_{i}^{\alpha} & =R_{i}^{\alpha, 1}+R_{i}^{\alpha, 2} \\
\eta_{i}^{\alpha} & =R_{i}^{\alpha, 3}
\end{aligned}
$$

Actually, $\eta_{i}^{\alpha}$ is the direction of the $i$ th ordered moment in Cartesian coordinates.

- Secondly, we consider only small deviations away from this ordered state.

As we shall see below, the spin wave theory is a field theory the quasi-particles of which are the so-called spin-waves or magnons. These excitations carry a spin one and correspond physically to precession modes of the spins around the directions $\vec{\eta}_{i}$ of the ordered moments and characterized by phase shift from site to site (see figure 5). The following paragraphs explain the mathematical formalism of the theory.

\subsection{Holstein-Primakov spin representation}

Owing to our second hypothesis, we have to describe small deviations from the ordered configuration. To achieve this we use a representation of the spin operators proposed by Holstein and Primakov. Introducing one boson operator $b_{m, i}$ per site, we have:

$$
\begin{aligned}
& S_{m, i}^{+} \approx \sqrt{2 S_{i}} b_{m, i} \quad S_{m, i}^{1} \approx \frac{\sqrt{2 S_{i}}}{2}\left(b_{m, i}+b_{m, i}^{+}\right) \\
& S_{m, i}^{-} \approx b_{m, i}^{+} \sqrt{2 S_{i}} \quad \text { and } S_{m, i}^{2} \approx \frac{\sqrt{2 S_{i}}}{2 i}\left(b_{m, i}-b_{m, i}^{+}\right) \\
& S_{m, i}^{3} \approx S_{i}-b_{m, i}^{+} b_{m, i}
\end{aligned}
$$

and we can write the Cartesian coordinates of the spin at site $(m, i)$ :

$$
S_{m, i}^{\alpha=x, y, z}=\frac{\sqrt{2 S_{i}}}{2} \bar{z}_{i}^{\alpha} b_{m, i}+\frac{\sqrt{2 S_{i}}}{2} z_{i}^{\alpha} b_{m, i}^{+}+\eta_{i}^{\alpha}\left(S_{i}-b_{m, i}^{+} b_{m, i}\right)
$$


In this bosonic representation the physical quantity is the number of bosons per site $b_{m, i}^{+} b_{m, i}$, which, according to second quantification, can take integer values between zero and infinity. It also allows a simple interpretation of the equation for $S_{m, i}^{3}$. Actually, the larger $b_{m, i}^{+} b_{m, i}$ the smaller the projection along the local axis $e_{i}^{3}, \vec{S}_{m, i}$ being thus mostly perpendicular to $e_{i}^{3}$. It is worth mentioning that this spin representation is valid only if the number of bosons remains small, so that the spin components perpendicular to $e_{i}^{3}$ remain small. Of course, in this context, "small" means small compared to the length of the spin $S_{i}$.

Turning back to the Heisenberg Hamiltonian, our problem is now written in the language of second quantification:

$$
\begin{aligned}
H=\sum_{m, n, i, j, \alpha, \beta} & \left\{\frac{\sqrt{2 S_{i}}}{2} \bar{z}_{i}^{\alpha} b_{m, i}+\frac{\sqrt{2 S_{i}}}{2} z_{i}^{\alpha} b_{m, i}^{+}+\eta_{i}^{\alpha}\left(S_{i}-b_{m, i}^{+} b_{m, i}\right)\right\} J_{m, i, n, j}^{\alpha \beta} \\
& \left\{\frac{\sqrt{2 S_{j}}}{2} \bar{z}_{j}^{\beta} b_{n, j}+\frac{\sqrt{2 S_{j}}}{2} z_{j}^{\beta} b_{n, j}^{+}+\eta_{j}^{\beta}\left(S_{j}-b_{n, j}^{+} b_{n, j}\right)\right\}
\end{aligned}
$$

Defining the $2 L$ vectors $X_{m}$ :

$$
X_{m}=\left(\begin{array}{c}
b_{m, 1} \\
\ldots \\
b_{m, i} \\
\ldots \\
b_{m, L} \\
b_{m, 1}^{+} \\
\ldots \\
b_{m, i}^{+} \\
\ldots \\
b_{m, L}^{+}
\end{array}\right)
$$

we finally obtain the following compact form:

$$
H=\sum_{m, n} X_{m}^{+} h_{m, n} X_{n}
$$

$h_{m, n}$ is a $2 L \times 2 L$ matrix whose elements are given by:

$$
\begin{array}{c|c|c} 
& j=1, \ldots, L & j=L+1, \ldots, 2 L \\
\hline i=1, \ldots, L & -\Omega_{m, n, i, j}+\frac{\sqrt{S_{i} S_{j}}}{2} z_{i} J_{m, i, n, j} \bar{z}_{j} & +\frac{\sqrt{S_{i} S_{j}}}{2} z_{i} J_{m, i, n, j} z_{j} \\
i=L+1, \ldots, 2 L & +\frac{\sqrt{S_{i} S_{j}}}{2} \bar{z}_{i} J_{m, i, n, j} \bar{z}_{j} & -\Omega_{m, n, i, j}+\frac{\sqrt{S_{i} S_{j}}}{2} \bar{z}_{i} J_{m, i, n, j} z_{j}
\end{array}
$$

and

$$
\Omega_{m, n, i, j}=\delta_{m, n} \delta_{i, j}\left(\sum_{\nu, \ell} S_{\ell} \eta_{i} J_{m, i, \nu, \ell} \eta_{\ell}\right)
$$

Of course, there is nothing new in this compact form, but it emphasizes the fact that we have to deal with a quadratic form. We are thus certain that it has an exact solution, which we shall find now. 


\subsection{Fourier transformation}

The "diagonalization" of this quadratic form can be carried out at least partly with a simple Fourier transformation. Defining the $2 L$ vectors $X_{k}=\sum_{m} e^{i k R_{m}} X_{m}$, we obtain:

$$
H=\sum_{k} X_{k}^{+} h_{k} X_{k}
$$

and $h_{k}$ is a $2 L \times 2 L$ matrix with elements:

\begin{tabular}{c|c|c} 
& $j=1, \ldots, L$ & $j=L+1, \ldots, 2 L$ \\
\hline$i=1, \ldots, L$ & $-\Omega_{i, j}+\frac{\sqrt{S_{i} S_{j}}}{2} z_{i} J_{k, i, j} \bar{z}_{j}$ & $+\frac{\sqrt{S_{i} S_{j}}}{2} z_{i} J_{k, i, j} z_{j}$ \\
$i=L+1, \ldots, 2 L$ & $+\frac{\sqrt{S_{i} S_{j}}}{2} \bar{z}_{i} J_{k, i, j} \bar{z}_{j}$ & $-\Omega_{i, j}+\frac{\sqrt{S_{i} S_{j}}}{2} \bar{z}_{i} J_{k, i, j} z_{j}$
\end{tabular}

and

$$
\Omega_{i, j}=\delta_{i, j}\left(\sum_{v, \ell} S_{\ell} \eta_{i} J_{m, i, v, \ell} \eta_{\ell}\right)
$$

\subsection{Bogolubov transformation}

We now have to diagonalise, for each $k$ wavevector, a $2 L \times 2 L$ matrix; but this is not strictly speaking a diagonalization. Actually, we have to define a basis transformation $P$ :

$$
X_{k}=P_{k} Y_{k}
$$

such that the Hamiltonian $H$ becomes diagonal:

$$
H=\sum_{k} Y_{k}^{+} E_{k} Y_{k}
$$

while the components $y_{k, \ell}$ of the $Y_{k}$ vector describe boson operators. This transformation is called the Bogolubov transformation.

In the language of field theory, the ground state is the vacuum of these operators, and the excited states contain a given number of particles. Physically, these particles are spin waves also called magnons, and correspond to precession modes of the ordered structure. As mentioned above, the validity of the model is controlled by monitoring the projection along $e_{i}^{3}, S_{i}-b_{m, i}^{+} b_{m, i}$ which must remain positive. Further, simple algebra shows that if there are $L$ magnetic ions, there are also $L$ spin wave branches, and the problem is now to find the matrix $P$ (the $k$ index is omitted for convenience).

The explicit formulation of the Bogolubov transformation, due to Wallace [8] is reproduced hereafter. It is based on the canonical bosonic commutation relations of the original b's operators:

$$
\left[X_{s}, X_{s^{\prime}}^{+}\right]=X_{s} X_{s^{\prime}}^{+}-X_{s^{\prime}}^{+} X_{s}=g_{s, s^{\prime}}
$$

where $g$ is a $2 L \times 2 L$ matrix, reading as

$$
g=\left(\begin{array}{ll}
I & \\
& -I
\end{array}\right)
$$

The solution is carried out in four steps, introducing new intermediate variables $Z_{1}, Z_{2}$ and $Z_{3}$. The first step is a standard diagonalization of $h$ :

$$
X=V Z_{1}
$$




$$
\left[Z_{1}, Z_{1}^{+}\right]=V^{+} g V
$$

which transforms the Hamiltonian into:

$$
H=Z_{1}^{+} T Z_{1}
$$

where $T$ is a diagonal matrix. The second step involves normalized operators $Z_{2}$ defined as:

$$
Z_{1}=T^{-1 / 2} Z_{2}
$$

with commutation relations:

$$
\left[Z_{2}, Z_{2}^{+}\right]=T^{1 / 2} V^{+} g V T^{1 / 2}=R
$$

The Hamiltonian thus becomes:

$$
H=Z_{2}^{+} Z_{2}
$$

The third step is based on a diagonalization of the $R$ matrix:

$$
W^{+} R W=E
$$

where $W$ is the matrix of the eigenvectors and $E$ the diagonal matrix of the eigenvalues. We then introduce the $Z_{3}$ operators:

$$
Z_{2}=W Z_{3}
$$

with commutation relations:

$$
\left[Z_{3}, Z_{3}^{+}\right]=W^{+} R W=E
$$

The solution now becomes clear, and the $Y$ operators are just a sort of normalized version of the $Z_{3}$ :

$$
Z_{3}=A Y
$$

with $A$ a diagonal matrix that remains to be explicated. As we impose bosonic commutation relations of the y's, we have $\left[Y, Y^{+}\right]=g$ and thus:

$$
\left[Z_{3}, Z_{3}^{+}\right]=A\left[Y, Y^{+}\right] A^{+}=A g A^{+}
$$

Owing to equation (3.1), we have the final results:

$$
A_{i}=\sqrt{\left|E_{i}\right|} \operatorname{sign}\left(E_{i}\right)
$$

and

$$
P=V T^{-1 / 2} W A
$$

If we are able to go through these steps, the initial problem is now fully solved. From a numerical point of view, the spin wave theory actually requires standard routines of linear algebra, and thus presents no difficulties.

\subsection{Spin-spin correlation function}

At the beginning of this lecture, we quoted that the neutron cross section is directly related to a set of spin-spin correlation functions:

$$
\left\langle S_{m, i}^{\alpha} S_{n, j}^{\beta}(t)\right\rangle
$$


In the framework of the Holstein-Primakov spin representation these functions can be easily calculated:

$$
\begin{aligned}
\left\langle S_{m, i}^{\alpha} S_{n, j}^{\beta}(t)\right\rangle= & \frac{\sqrt{S_{i} S_{j}}}{2}\left\langle\left(b_{m, i}^{+} b_{m, i}\right)\left(\begin{array}{ccc}
z_{i}^{\alpha} \bar{z}_{j}^{\beta} & z_{i}^{\alpha} & z_{j}^{\beta} \\
\bar{z}_{i}^{\alpha} \bar{z}_{j}^{\beta} & \bar{z}_{i}^{\alpha} & z_{j}^{\beta}
\end{array}\right)\left(\begin{array}{l}
b_{n, j}(t) \\
b_{n, j}^{+}(t)
\end{array}\right)\right\rangle \\
& +\eta_{i}^{\alpha} \eta_{j}^{\beta}\left(S^{2}-S\left\langle b_{m, i}^{+} b_{m, i}+b_{n, j}^{+} b_{n, j}\right\rangle\right)
\end{aligned}
$$

Making use of the Bogolubov transformation, we can write the $b$ 's as a function of the $y$ 's. Moreover, as the $y_{k, \ell}$ are bosons, we have

$$
\begin{aligned}
& \left\langle y_{k, \ell}^{+} y_{k^{\prime}, \ell^{\prime}}(t)\right\rangle=\delta_{k, k^{\prime}} \delta_{\ell, \ell^{\prime}} n\left(E_{k, \ell}\right) e^{-i E_{k, \ell} t} \\
& \left\langle y_{k, \ell} y_{k^{\prime}, \ell^{\prime}}^{+}(t)\right\rangle=\delta_{k, k^{\prime}} \delta_{\ell, \ell^{\prime}}\left(1+n\left(E_{k, \ell}\right)\right) e^{+i E_{k, \ell} t}
\end{aligned}
$$

where $n(E)$ is the Bose distribution at temperature $T$ :

$$
n(E)=\frac{1}{e^{E / T}-1}
$$

We finally obtain the following formal expression

$$
\begin{aligned}
\frac{\partial^{2} \sigma}{\partial \Omega \partial E_{f}}(Q, \omega) \propto & \sum_{k, \tau} \delta(Q+k-\tau) \sum_{\ell} \\
& n\left(E_{-k, \ell}\right) \delta\left(\omega+E_{-k, \ell}\right)\left(\sum_{i, j} \frac{\sqrt{S_{i} S_{j}}}{2} e^{i Q\left(u_{i}-u j\right)} A_{i, j, \ell}(-k)\right) \\
& +\left(1+n\left(E_{k, \ell}\right)\right) \delta\left(\omega-E_{k, \ell}\right)\left(\sum_{i, j} \frac{\sqrt{S_{i} S_{j}}}{2} e^{i Q\left(u_{i}-u j\right)} B_{i, j, \ell}(k)\right)
\end{aligned}
$$

where the $A$ and $B$ quantities have to be computed numerically and depend on the basis transformation matrix $P$. Note that the exact positions of the spins $u_{i}$ within the unit cell also appear in the formulas. The important point is that the cross section actually contains a series of Dirac functions centred at $\omega= \pm E_{k, \ell}$, corresponding to the creation and annihilation of magnons. The remainder is nothing but a dynamical structure factor. In other terms, as long as this structure factor is not too small, a neutron experiment gives access to their dispersion.

\subsection{Example}

Let us now take an example and consider the particular case of spins on a triangular lattice. This model orders at zero temperature in a standard 120 degree configuration, as shown in figure 6 . As we have 3 spins per magnetic unit cell, we expect 3 spin wave modes. Figure 6 shows a mapping of the dynamical structure factor as a function of $Q$ taken along the reciprocal direction $a^{*}$. We observe the expected 3 delicate arches, and we note that they have different intensities. If we move to another zone centre, for instance around (006), these intensities are strongly affected, while the dispersions remain identical. This result highlights one of the relevant points of such a calculation. Indeed, a well-informed neutron user would take advantage of these numerical results and carry out the corresponding experiment in the proper Brillouin zone, enhancing the signal, and extracting the dispersions under good conditions.

Let us now consider the effect of a planar anisotropy term in the Hamiltonian, the triangular plane being the easy plane. As shown in figure $7 \mathrm{a}$, it induces a gap at the zone centre. This gap corresponds to the fact that the precession of the spins must now overcome a new energetic barrier, moving the spins 

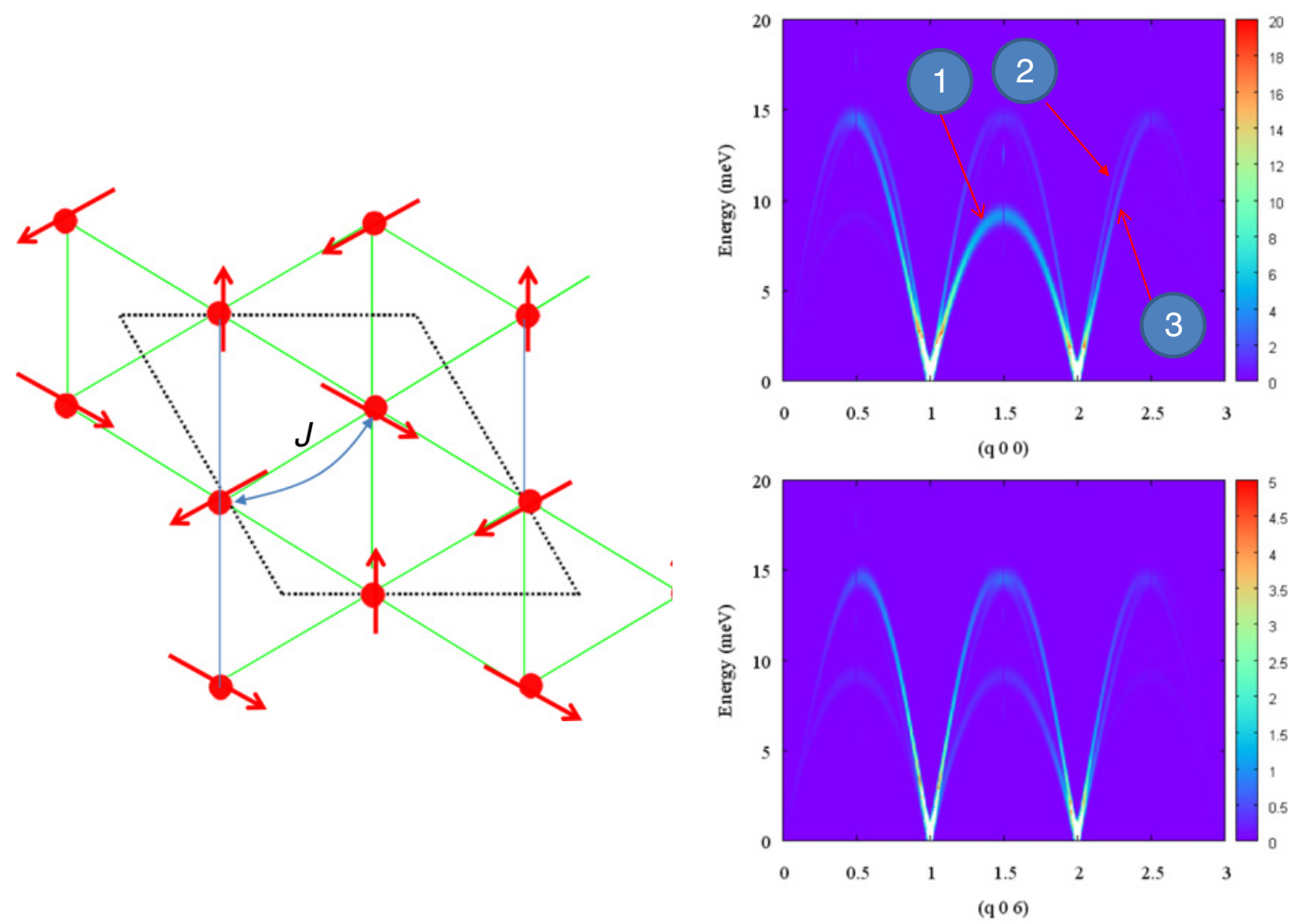

Figure 6. (left) Schematic diagram of the triangular lattice. (right) Mapping of the spin-spin correlation function measured as a function of energy and $Q$ taken along $a^{*}$. Three spin wave branches are visible, the intensity of which depends on the zone centre (100) or (006).

out of the easy plane. Interestingly, there is one remaining mode the energy of which goes to zero at the zone centres. This is the Goldstone mode of the magnetic structure.

To further explain the spin wave dynamics in the triangular lattice, we can calculate other correlation functions, for instance, between spin components within the easy plane (figure 7b) or spin components along the c-axis (perpendicular to the easy plane, figure 7c ). In agreement with previous considerations, we observe that the Goldstone mode is indeed due to the former. Note that a polarized neutron experiment would measure these new correlation functions, thus providing more information, but of course at the expense of beam time. In the present case, the numerical simulation is thus helpful and allows understanding the different contributions which are mixed together in a standard experiment.

\subsection{Beyond spin waves}

In this first section, we have seen that the dynamics of spin waves can be routinely measured by inelastic neutron scattering. The theory is actually a "simple" theory of free bosons, while the correlation function can be calculated with relatively simple numerical simulations, based on linear algebra. But this is not a panacea. Actually, a number of magnets never order, owing to strong quantum fluctuations, low dimensionality and frustration effects.

For instance, recent progress in the physics of low dimensional systems has shown that the $S=1$ elementary magnetic excitation, the spin wave, is made of two spin-half quantum entities called spinons, which under usual circumstances remain confined within the magnon and thus are not visible $[9,10]$. 

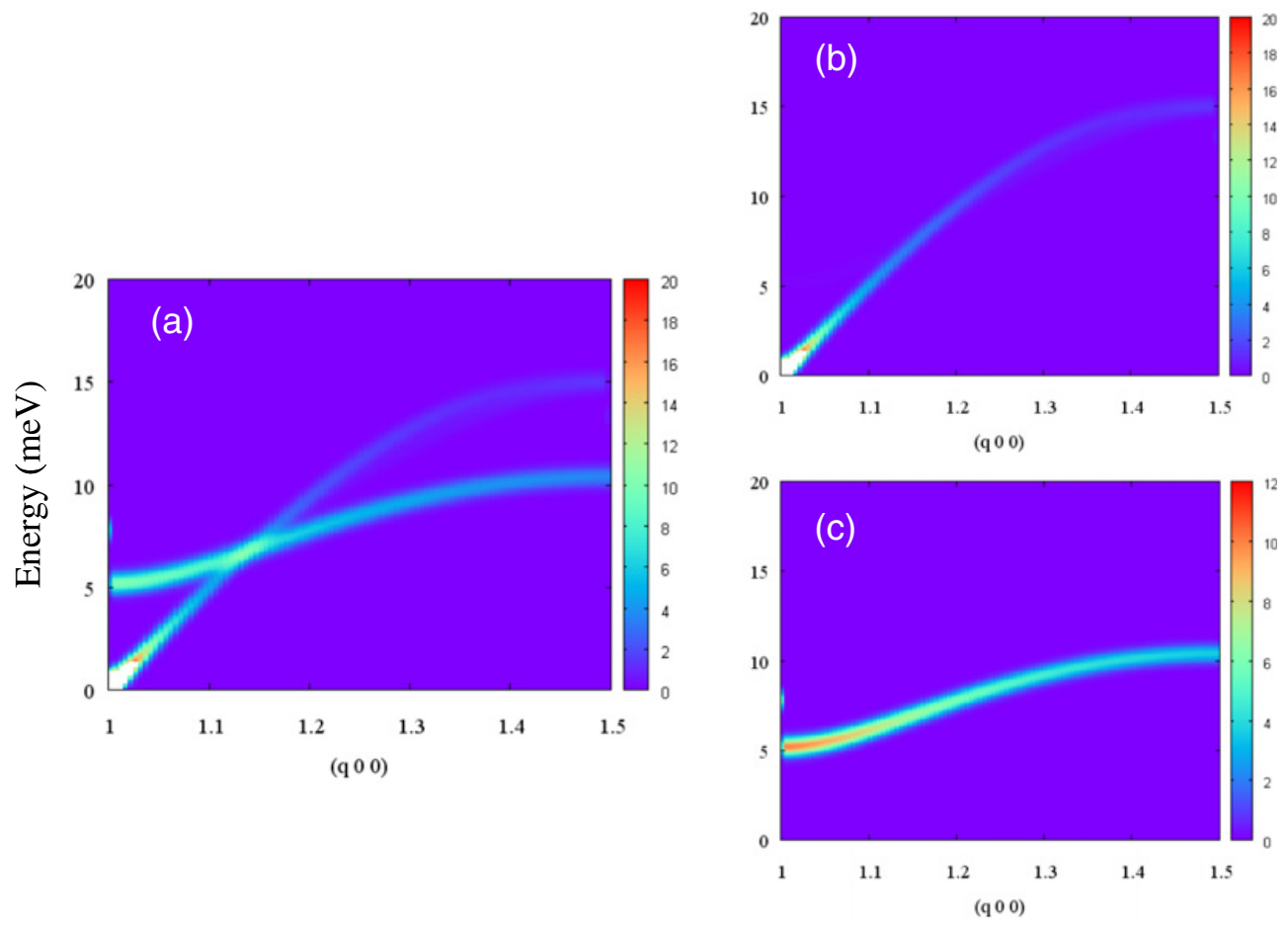

Figure 7. (a) Influence of a planar anisotropy term on the spin-spin correlation function. Two branches are now characterized by a spin gap at the zone centre. (b) and (c) show respectively the correlation function between spin components within the $(\mathrm{ab})$ plane and along the c-axis.

Actually, this concept of confinement, when particles are bound together by an interaction whose strength increases with increasing particle separation, is a fundamental idea of modern physics today.

The most widely studied model in which this phenomenon takes place is the standard spin-half Heisenberg antiferromagnetic chain (1D) with first-neighbour interactions $\left(J_{1}\right)$ [11]. In agreement with the Wagner and Mermin theorem, quantum fluctuations prevent static long-range order and the ground state remains a spin liquid, with power-law antiferromagnetic correlations. In such a chain, spinons can be seen as domain walls between reversed antiferromagnetic domains; they can move freely and independently along the chain, as no energy cost is involved: in other words, they do not confine. In this case, the dynamical structure factor shows no coherent excitation but an incoherent spectrum called two-spinon continuum, which reflects the range of energies corresponding to the different spinon pairs. Experimental evidence of the two-spinon continuum has been recently reported in quasi-1D systems such as $C u P z N$ [12] or $C D C$ [13], and the quantum spin ladder $\left(C_{5} D_{12} N\right)_{2} C u B r_{4}$ [14], as well as in more complex spin-half compounds like the square ladder $\mathrm{CaCu}_{2} \mathrm{O}_{3}$ [15], or the two-dimensional triangular lattice $\mathrm{Cs}_{2} \mathrm{CuCl}_{4}[16]$.

Even if the spin wave theory is one of the milestones in magnetism, this example shows that it is not sufficient to describe the large range of exotic phenomena such as the spinon de-confinement. New theoretical concepts along with numerical calculations have to be developed to describe them.

\section{CLASSICAL SPIN DYNAMICS}

Among these developments, we propose to detail in this section the particular case of classical dynamics, which has the drawback of neglecting quantum effects, but the advantage of addressing the problem 
of fluctuating or disordered (spin liquid) magnets, along with an exact solution [17-20]. It involves Monte Carlo simulations, and shares common trends with other approaches, as for instance molecular dynamics. Thus we still consider the Heisenberg Hamiltonian

$$
H=\sum_{i, j} \vec{S}_{i} J_{i, j} \vec{S}_{j}
$$

but we shall now assume that the spin is a classical variable. As a result, the equation of motion simply reads as:

$$
\frac{d}{d t} \vec{S}_{i}=\vec{S}_{i} \times \sum_{j} J_{i, j} \vec{S}_{j}
$$

and this (non linear) differential equation is the starting point of the numerical simulations. In this framework, we can calculate a number of physical quantities such as relaxation of the system, life times, interactions between modes, emphasizing non linear effects. But before going further, we have to explain the basis of the Monte Carlo method.

\subsection{Monte Carlo}

This method is actually devoted to calculating integrals. If we want to calculate

$$
I=\int_{a}^{b} d x f(x)
$$

we can find an approximate value by computing

$$
I \approx \frac{b-a}{N} \sum_{i=1, N} f\left(x_{i}\right)
$$

where the $x_{i}$ are uniformly (randomly) distributed in the $[a, b]$ interval. Of course, the uniform distribution may not be very well suited for our problem. For instance, it can be much more convenient not to spend too much time in calculating $f(x)$ in regions where $f(x)$ is zero! To achieve this, there is a standard biasing technique which consists in sampling $f(x)$ in "important regions". By simply considering that

$$
I=\int d x \frac{f(x)}{P(x)} P(x)
$$

then, if the $x_{i}$ are sampled according to a distribution $P(x)$ we simply write

$$
I \approx \frac{1}{N} \sum_{i=1, N} \frac{f\left(x_{i}\right)}{P\left(x_{i}\right)}
$$

At this stage, it is worth noting that the correlation functions (and expectation values) are indeed integrals over a set of spin configurations:

$$
\langle O\rangle_{T}=\frac{1}{Z} \int d x O(x) e^{-\beta H(x)}
$$

Here $O$ is the "observable", $Z$ the partition function and $x$ denotes the spin configuration. It is clearly of the previous form if

$$
f(x)=O(x) e^{-\beta H(x)}
$$

The question we shall now answer is: what is the $P$ distribution we should use? It is very tempting to choose

$$
P(x)=e^{-\beta H(x)}
$$


as in that case:

$$
\langle O\rangle_{T} \approx \frac{1}{N} \sum_{i=1, N} O\left(x_{i}\right)
$$

However, we now have to sample $x_{i}$ according to $P(x)=e^{-\beta H(x)}$ and this can be quite tricky.

\subsection{Metropolis sampling}

This sampling is achieved with the Metropolis algorithm. It is not the purpose of this lecture to enter into much details, but it is important to mention that it relies on the Markov process notion. This procedure generates a series of configurations $x$, the evolution from iteration $x$ to iteration $y$ being determined by a probability transition $W(x \rightarrow y)$. This probability is further characterized by the following properties:

$$
\begin{aligned}
W(x \rightarrow y) & \geq 0 \\
\sum_{y} W(x \rightarrow y) & =1 \\
P(x) W(x \rightarrow y) & =P(y) W(y \rightarrow x)
\end{aligned}
$$

The second property ensures that all configurations can be reached starting from $x$, while the third one that the process is reversible in agreement with the detailed balance principle. After a sufficient number of iterations, a stationnary state is reached, that verifies

$$
\frac{W(x \rightarrow y)}{W(y \rightarrow x)}=\frac{P(y)}{P(x)}
$$

With the choice $P(x)=e^{-\beta H(x)}$, the previous equation reads as:

$$
\frac{W(x \rightarrow y)}{W(y \rightarrow x)}=e^{-\beta(H(y)-H(x))}
$$

Taking advantage of these developments, the Metropolis algorithm generates random configurations according to the distribution $P(x)$ :

- Consider a configuration $x$

- Generate a random configuration $y$

- If $H(x) \leq H(y)$ or if $p \leq \exp -H(x) / T$ where $p$ is a uniform random number in $[0,1]$ then $y$ is accepted as the new current configuration; if not, $y$ is rejected.

- Repeat many times to reach the stationary state.

\subsection{Putting things together...}

We now have an efficient method to sample the spin configurations according to the canonical distribution. It is thus easy to calculate integrals:

$$
\langle O\rangle_{T} \approx \frac{1}{N} \sum_{i=1, N} O\left(x_{i}\right)
$$

To determine the classical dynamics, and calculate the spin-spin correlation, we thus have to compute the integral:

$$
\left\langle S_{m} S_{n}(t)\right\rangle_{T} \approx \frac{1}{N} \sum_{i=1, N} S_{m}\left(t=0, x_{i}\right) S_{n}\left(t, x_{i}\right)
$$


(a)

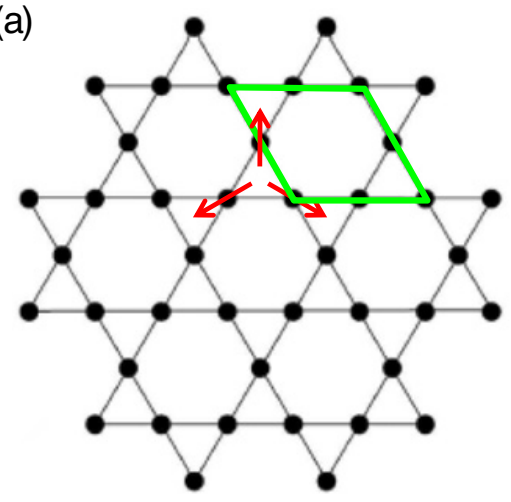

(c)

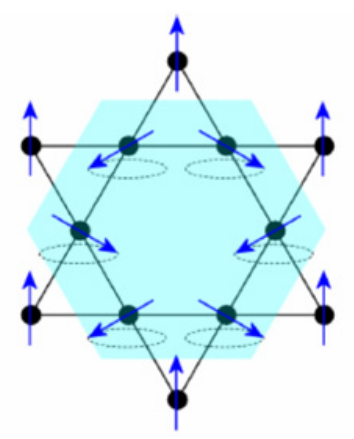

(b)

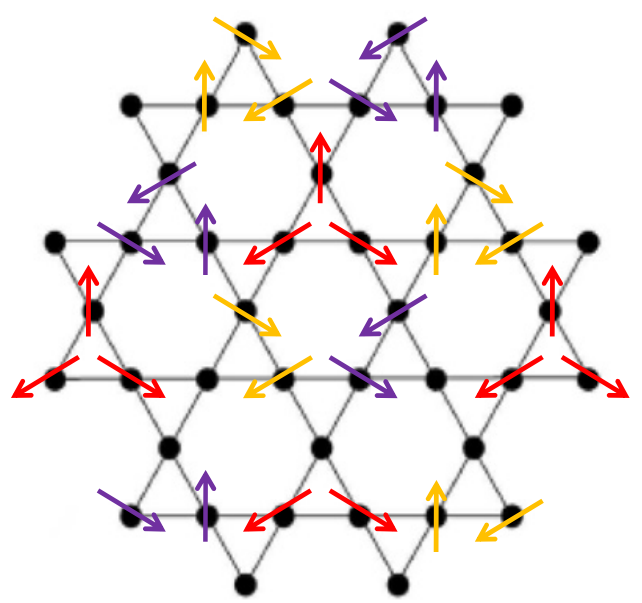

Figure 8. (a) Kagome lattice, (b) schematic diagram of the $\sqrt{3} \times \sqrt{3}$ spin configuration and (c) sketch of the so called "soft mode", corresponding to rotations of the spins belonging to a hexagon.

The value of the spin at time $t$ is determined from the numerical integration of the equation of motion, in close analogy with molecular dynamics:

$$
\frac{d}{d t} \vec{S}_{i}=\vec{S}_{i} \times \sum_{j} J_{i, j} \vec{S}_{j}
$$

Finally, the program is the following:

- Generate $N$ spin configurations according to $P(x)=\exp -H(x) / T$

- For each, integrate the differential equation

- Calculate the desired expectation values.

\subsection{Example}

To illustrate this method, let us now comment on the recent results obtained by Robert et al. [19] on the Kagome lattice (see figure 8a). The ground state of that model is macroscopically degenerate: the system keeps fluctuating and never orders. Actually, two regimes can be distinguished, with, at high temperature, the exponential spin liquid regime, and at low temperature the planar algebraic regime. The emergence of the latter is explained by an order-by-disorder mechanism. This means that even if the ground state is degenerate, the system can minimize its free energy if the spins are co-planar.

Figure 9a shows a colour map of the spin-spin correlation function as a function of energy and wavevector taken along $\mathrm{a}^{*}$. At low temperature, we observe propagating modes, even if the system has no long range order; two branches are visible, an acoustic and an optical one. At very low energy, extra 
(a)

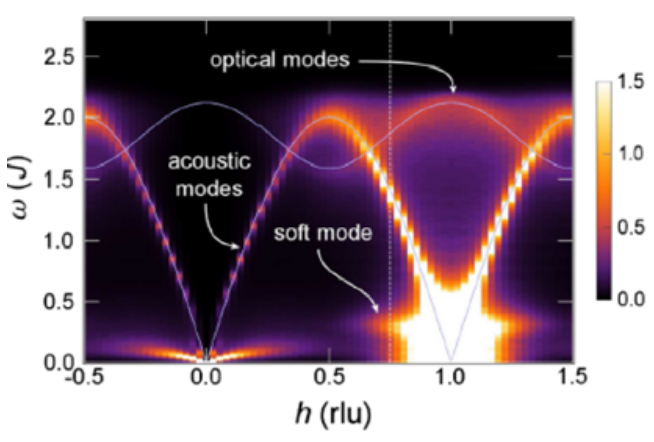

(d)

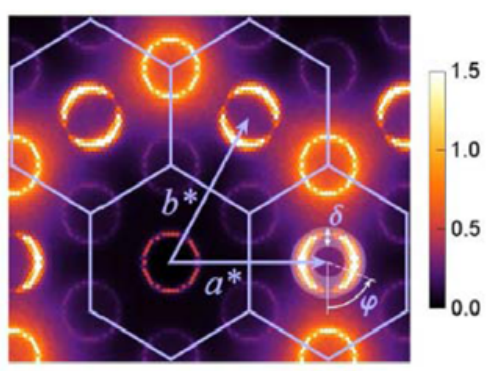

(b)

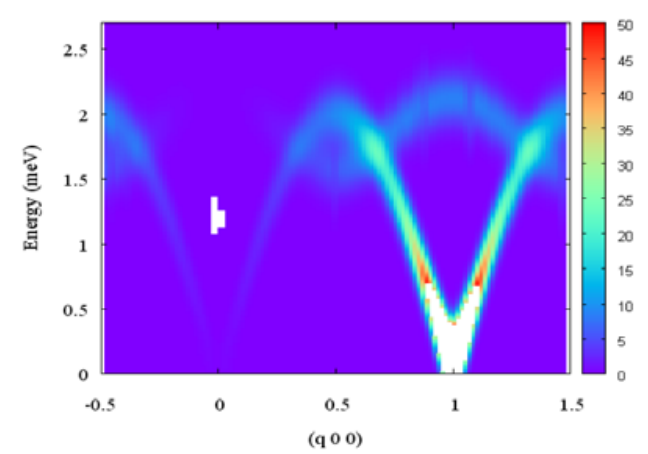

(c)

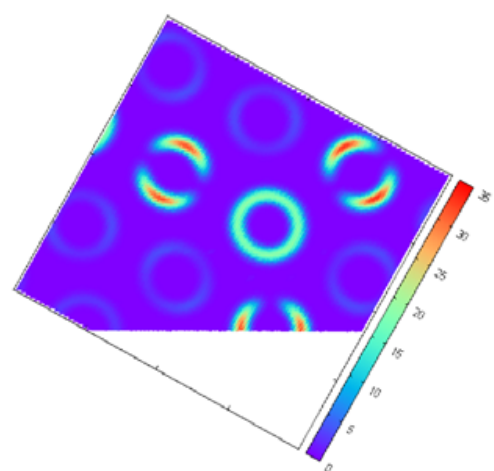

Figure 9. Colour mapping of the spin-spin correlation function: (a) and (b) are calculated within the Monte Carlo approach and spin wave theory respectively [19]. The two top mappings are calculated along $a^{*}$. The bottom mappings are energy cuts at a constant energy while the wavevector varies in the $\left(a^{*}, b^{*}\right)$ plane.

weakly dispersing intensity appears. This feature is the signature of a so called "soft mode" which results from the degeneracy of the system. It corresponds to rotations of the spins belonging to a hexagon, as sketched in figure 8c. A constant energy map, shown in figure 9d, allows to observe a cut across the conical dispersion of the acoustic mode emerging from the reciprocal lattice nodes. The intensity of some of them shows remarkable "half moon" shape, which is due to an interference phenomenon specific to the triangular geometry.

At this point, it is interesting to wonder what we would have obtained in the framework of the spin wave theory. As the system does not order, it is in principle not possible. However, we note that among the spin configuration, it turns out that the $\sqrt{3} \times \sqrt{3}$ pattern (see 8b) is favoured, meaning that the system is most of the time in that particular configuration, even if it is still fluctuating. Hence, we will assume that the system orders in that particular configuration and calculate the spin wave dispersion along with the spin-spin correlation function. The result is shown in figure $9 \mathrm{~b}$ and $9 \mathrm{c}$. Interestingly, the two branches also appear, exhibiting the same "half moon" shape; however, important features cannot be captured by the model, as for instance the "soft mode". Instead, the spin wave theory predicts a zero energy mode, which is not visible on the figure, but is indeed nothing but a precursor of the soft mode.

In summary, the classical spin dynamics approach provides exact results, neglecting quantum aspects. For instance, it is valid for large $\mathrm{S}$ only. Compared to the spin wave theory, it allows to go a step further, shedding light on important features of exotic models as for instance the Kagome lattice Heisenberg Hamiltonian. It allows understanding new phenomena, as in that particular case the existence and properties of the soft mode. 


\section{SUMMARY}

In summary, we have detailed two different numerical approaches that allow the study of spin dynamics in magnets. The first one, the spin wave theory, is one of the milestones in magnetism. While quite simple, based on standard linear algebra, it can become quite tricky for incommensurate and/or complex systems. In contrast, the classical spin dynamics allows to eliminate a number of restrictive hypotheses of the spin wave theory, allowing to address new questions. It is based on the numerical integration of a differential equation, embedded in a Monte Carlo calculation of the expectation values (Metropolis algorithm).

With the recent development of computers, these simulations gradually become accessible to experimentalists. Used with care, they are helpful to prepare or interpret the results of a neutron scattering experiment.

\section{References}

[1] P.W. Anderson, Phys. Rev. 83, 1260 (1951).

[2] R. Kubo, Phys. Rev. 87, 568 (1952).

[3] T. Oguchi, Phys. Rev 117, 117 (1960).

[4] C. Tsallis, Diagonalization method for the general Hamiltonian of an assembly of bosons, J. Math. Phys, 19, 277 (1978).

[5] D.C. Mattis, Theory of Magnetism I, Springer Verlag, 1988.

[6] R.M. White, Quantum Theory of Magnetism, Springer Verlag, 1987.

[7] A. Auerbach, Interacting electrons and Quantum Magnetism, Springer Verlag, 1994.

[8] D.C. Wallace, PR, 128, 1614 (1962) Spin waves in complex lattice.

[9] L. D. Faddeev and L. A. Takhtajan, Physics Letters A,85, 375 (1981).

[10] G. Miller, H. Thomas, H. Beck, and J. C. Bonner, Physical Review B, 24, 1429 (1981).

S. Sachdev, Physical Review B, 45, 12377 (1992).

[11] J. Cloizeaux and J. J. Pearson, Physical Review, 128, 2131 (1962).

[12] M. B. Stone, D. H. Reich, C. Broholm, K. Lefmann, C. Rischel, C. P. Landee, and M. M. Turnbull, Physical Review Letters, 91, 037205 (2003).

[13] M. Kenzelmann, Y. Chen, C. Broholm, D. H. Reich, and Y. Qiu, Physical Review Letters, 93, 017204 (2004).

[14] B. Thielemann, C. Ruegg, H. M. Ronnow, A. M. Lauchli, J. S. Caux, B. Normand, D. Biner, K. W. Kramer, H. U.Gudel, J. Stahn, K. Habicht, K. Kiefer, M. Boehm, D. F McMorrow, and J. Mesot, Physical Review Letters, 102, 107204 (2009).

[15] B. Lake, A. M. Tsvelik, S. Notbohm, D. A. Tennant, T. G. Perring, M. Reehuis, C. Sekar, G. Krabbes, and B. Buchner, Nature Physics, 6, 50 (2010).

[16] R. Coldea, D. A. Tennant, A. M. Tsvelik, and Z. Tylczynski, Physical Review Letters, 86, 1335 (2001).

[17] Xiupin Tao et al., PRL 95, 087207 (2005).

[18] Conlon and Chalker, PRL 102, 237206 (2009).

[19] Robert et al., PRL 101, 117207 (2008).

[20] G. Mueller, PRL 60, 2785 (1988). 